\title{
AVALIAÇÃO DE DIFERENTES MÉTODOS DE EXTRAÇÃO PARA A IDENTIFICAÇÃO DE RESÍDUOS DE MACROLÍDEOS EM ALIMENTOS INFANTIS INDUSTRIALIZADOS À BASE DE CARNE POR CROMATOGRAFIA A LÍQUIDO ACOPLADA À ESPECTROMETRIA DE MASSAS SEQUENCIAL (LC-MS/MS)
}

\author{
Jônatas V. Grutes*,a, Rosana G. Ferreira ${ }^{\mathrm{a}}$, Mychelle A. Monteiro ${ }^{\mathrm{a}}$, Mararlene U. Pereira ${ }^{\mathrm{a}}$ e Bernardete F. Spisso ${ }^{\mathrm{a}}$ \\ anstituto Nacional de Controle de Qualidade em Saúde, Fundação Oswaldo Cruz, Rio de Janeiro - RJ, Brasil
}

Recebido em 02/03/2018; aceito em 01/10/2018; publicado na web em 07/11/2018

\begin{abstract}
EVALUATION OF DIFFERENT EXTRACTION METHODS FOR IDENTIFICATION OF MACROLIDES RESIDUES IN MEATBASED CANNED BABY FOOD BY LIQUID CHROMATOGRAPHY-TANDEM MASS SPECTROMETRY (LC-MS/MS). The interest in a better quality of life raised the concern about food safety. The administration of veterinary drugs in livestock may lead the occurrence of residues in food that may be harmful to consumers. Processed products are being consumed more and more, which increases concern for the safety of infants and children because they are more susceptible physiologically. Among these products stand out canned baby foods, defined by Ordinance 34/1998 issued by Brazilian Ministry of Health. Eggs and meat-based salty products for infants and children, legally designated as soups, fit into this definition and can not contain residues of hormones, antibiotics, as well as other pharmacologically active substances. Several techniques are used as identification and confirmatory assays of veterinary drug residues in food, although the main used technique is liquid chromatography - tandem mass spectrometry (LC-MS/MS). The aim of this work was to make a comparative assessment between different extraction methods in order to establish the best condition within the tested ones for the identification of macrolide residues in meat-based canned baby food by LC-MS/MS. A simple and effective extraction method, with good recoveries (65-93\%) and satisfactory relative standard deviations (0.4-11\%), was achieved using QuEChERS procedure for sample preparation.
\end{abstract}

Keywords: residues; antimicrobials; macrolides; baby food; mass spectrometry.

\section{INTRODUÇÃO}

Nos dias de hoje é cada vez maior a preocupação com a segurança dos alimentos, sobretudo devido à busca da sociedade por uma melhor qualidade de vida. ${ }^{1}$ Sabe-se que os medicamentos veterinários ocupam papel significativo no incremento da produção no setor pecuário. ${ }^{2}$ Entretanto, a contaminação química devido à essa utilização pode se tornar um grande problema para a saúde dos consumidores devido à presença dos resíduos de medicamentos veterinários, definidos como todos os compostos ou seus metabólitos presentes em qualquer porção comestível de um produto de origem animal. ${ }^{3,4}$ Diversos órgãos de regulamentação estabelecem Limites Máximos de Resíduos (LMR) permitidos para alimentos. Concentrações acima desses LMR para substâncias autorizadas e a presença de substâncias proibidas podem elevar as chances do desenvolvimento de reações alérgicas, discrasias sanguíneas e carcinogenicidade nos consumidores. Além disso, no caso dos antimicrobianos, mesmo concentrações abaixo desses limites podem induzir o surgimento de bactérias resistentes. ${ }^{5}$ Ressalta-se que os efeitos prejudiciais à saúde anteriormente citados podem ser ainda mais pronunciados em lactentes (crianças de zero a doze meses incompletos) e crianças de primeira infância (crianças de um a três anos), ${ }^{6}$ tanto por serem mais suscetíveis fisiologicamente, quanto por estarem, proporcionalmente, mais expostos aos alimentos do que adultos, levando-se em consideração a taxa de consumo por unidade de peso. ${ }^{7}$ Inseridos no contexto dos antimicrobianos de uso veterinário, os macrolídeos se destacam devido à sua ampla utilização na pecuária no tratamento de micoplasmose, doença digestiva hemorrágica, infecções respiratórias e abscessos hepáticos, e por serem classificados como criticamente importantes para a indução do surgimento de bactérias resistentes pela Organização Mundial da Saúde e Organização Mundial de Saúde Animal. ${ }^{8,9}$ Os macrolídeos

*e-mail: jvgrutes@gmail.com se caracterizam por apresentarem em suas estruturas químicas um anel lactona macrocíclico contendo 14 (eritromicina, roxitromicina, claritromicina, oleandomicina e troleandomicina), 15 (azitromicina) ou 16 (espiramicina, tilosina e tilmicosina) átomos de carbono, com moléculas de açúcares ligadas por ligações glicosídicas. ${ }^{10}$ A Figura 1 apresenta as estruturas químicas da tilosina e da espiramicina, respectivamente, dois dos principais macrolídeos empregados na medicina veterinária.
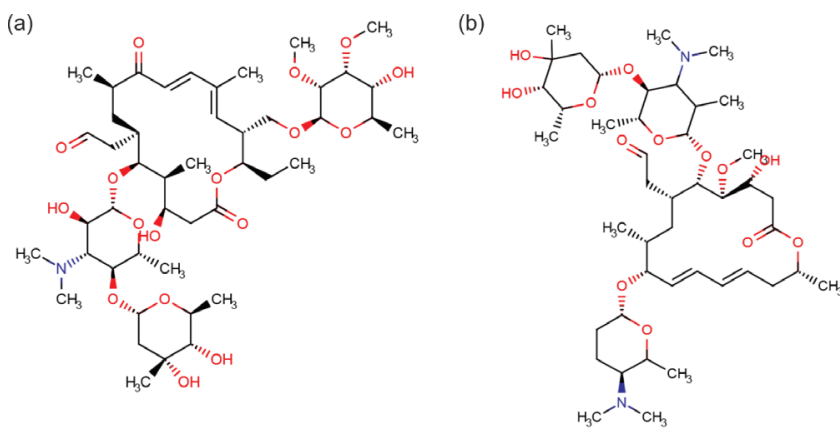

Figura 1. Estrutura química de dois dos principais macrolídeos empregados na medicina veterinária. (a) tilosina, (b) espiramicina ${ }^{l 1}$

Quanto à regulação de resíduos em alimentos infantis, especificamente em alimentos de transição, ou seja, aqueles industrializados utilizados como complemento do leite materno e que tem como objetivo promover uma adaptação progressiva aos alimentos comuns, a Portaria n 34 de 1998 explicita que não é admissível a presença de resíduos de hormônios, antibióticos, bem como resíduos de substâncias farmacologicamente ativas em tais produtos. Enquadram-se nessa definição os alimentos utilizados como refeições salgadas à base de carnes e ovos para lactentes e crianças de primeira infância, legalmente designados como sopinhas. ${ }^{6}$ A aplicação do conceito de 
tolerância zero exige que sejam empregados nas análises métodos com alta sensibilidade e, devido à complexidade e variedade das matrizes desses alimentos, um processo adequado de extração das amostras se faz necessário. ${ }^{12}$ As principais dificuldades analíticas na análise de resíduos como, por exemplo, os baixos níveis de concentração e o grande número de substâncias pesquisadas podem ser superados com o emprego da técnica analítica adequada, em que se destaca a cromatografia a líquido acoplada à espectrometria de massas sequencial (LC-MS/MS). ${ }^{13}$ Entretanto, uma matriz tão complexa, como as sopinhas, requer procedimentos de preparo de amostras apropriados que garantam a desproteinização, a remoção de gordura e açúcares e a extração dos fármacos, evitando interferências da matriz na análise e permitindo a aplicação da legislação relativa a esses alimentos. ${ }^{10,14}$ Além disso, a combinação entre uma técnica de extração simples e o uso da LC-MS/MS possibilita que o método seja mais rápido e econômico que outros. ${ }^{7}$ Nesse contexto, o método QuEChERS (Quick, Easy, Cheap, Effective, Rugged Safe) para extração e limpeza de extratos atraiu grande interesse nos últimos anos. Originalmente baseado na extração/partição prévia da amostra utilizando acetonitrila, sulfato de magnésio e cloreto de sódio com posterior etapa de limpeza empregando extração por fase sólida dispersiva combinando amina primária e secundária e sulfato de magnésio. ${ }^{15}$ Este método apresenta vantagens sobre métodos tradicionais de preparo de amostra por proporcionar altos percentuais de recuperação para um grande número de substâncias de diferentes polaridades e volatilidades. Além disso, permite a realização de determinações rápidas com pequenos volumes de solventes e pode ser realizado por um único analista, sem a necessidade de utilização de muitos materiais e equipamentos. ${ }^{16}$ Pelas vantagens e número de estudos que o utilizam pode-se afirmar que o método QuEChERS representa o estado da arte na determinação multirresíduos em alimentos. ${ }^{17}$ Embora ainda em pequeno número, pesquisas mostraram ser possível a determinação com sucesso de diversos medicamentos veterinários em alimentos de transição por LC-MS. ${ }^{7,18} \mathrm{O}$ objetivo desse trabalho foi realizar uma avaliação comparativa entre diferentes métodos de extração, com o intuito de se estabelecer a melhor condição entre os testados, para a obtenção de um método qualitativo de identificação de resíduos de macrolídeos em alimentos industrializados à base de carne por LC-MS/MS.

\section{PARTE EXPERIMENTAL}

\section{Amostras}

As amostras dos alimentos infantis (sopas) foram adquiridas em redes de supermercados e farmácias da cidade do Rio de Janeiro e armazenadas em temperatura ambiente.

\section{Padrões e reagentes}

Os padrões analíticos de estearato de eritromicina (ERI), tartarato de tilosina (TIL) e troleandomicina (TROL) foram obtidos da Farmacopeia Americana (Rockville, EUA). Com exceção da TROL (83\% de pureza) as substâncias possuíam purezas maiores que $99 \%$. A espiramicina (ESPI) foi adquirida da Sigma-Aldrich (St. Louis, EUA) e possuía pureza de 87,7\%. A claritromicina (CLA) (100\% de pureza) foi obtida da Farmacopeia Brasileira (Santa Maria, Brasil). A oleandomicina (OLE), a tilmicosina (TILM) e a roxitromicina (ROX), todas como purezas superiores a 96,5\%, foram adquiridas da Dr. Ehrenstorfer (Augsburg, Alemanha), sendo a ROX escolhida como padrão interno. Os reagentes acetato de sódio anidro (NaOAc), acetonitrila (ACN) para cromatografia a líquido, ACN para LC-MS, ácido acético (HOAc), ácido fórmico (FOA), carbonato de potássio $\left(\mathrm{K}_{2} \mathrm{CO}_{3}\right)$, cloreto de sódio $(\mathrm{NaCl})$, sulfato de magnésio $\left(\mathrm{MgSO}_{4}\right)$ e sulfato de sódio $\left(\mathrm{Na}_{2} \mathrm{SO}_{4}\right)$ foram adquiridos da Merck (Darmstadt, Alemanha) e possuíam purezas superiores a 99\% com exceção do FOA (98-100\%). O metanol (MeOH) para cromatografia a líquido foi obtido na J. T. Baker (Phillipsburg, EUA) e possuía pureza superior a 99,8\%. A amina primária e secundária (PSA) foi adquirida da Agilent Technologies (Santa Clara, EUA) e possuía pureza superior a $99 \%$. A água purificada tipo I foi obtida por sistema Milli-Q (Millipore, Bedford, EUA).

\section{Soluções estoque e intermediárias}

Cada padrão foi pesado e solubilizado em $\mathrm{MeOH}$ a fim de se obter soluções padrão estoques com concentrações de aproximadamente $1000 \mu \mathrm{g} \mathrm{mL}^{-1}$. As soluções foram transferidas para microtubos e armazenadas em temperaturas inferiores a $-70^{\circ} \mathrm{C}$. Para a fortificação das amostras foram preparadas soluções padrão intermediárias contendo um mix dos analitos a partir das soluções padrão estoques. As soluções foram preparadas visando atingir concentrações finais dos extratos iguais em todos os métodos. As concentrações das soluções padrão intermediárias encontram-se descritas na Tabela 1 .

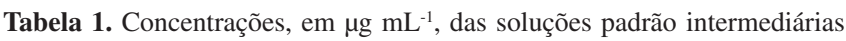
utilizadas nos experimentos

\begin{tabular}{cc}
\hline Soluções padrão intermediárias & $\begin{array}{c}\text { Concentração } \\
\left(\mu \mathrm{g} \mathrm{mL} \mathrm{mL}^{-1}\right)\end{array}$ \\
\hline $\begin{array}{c}\text { Padrão interno } \\
\text { (fortificação antes da extração - todos os métodos) }\end{array}$ & 2,00 \\
\hline $\begin{array}{c}\text { Mix de macrolídeos } \\
\text { (fortificação antes da extração - todos os métodos) }\end{array}$ & 2,00 \\
\hline $\begin{array}{c}\text { Mix de macrolídeos } \\
\text { (fortificação após a extração - métodos 1, 2, 3, 4, 6 e 8) }\end{array}$ & 1,50 \\
\hline $\begin{array}{c}\text { Mix de macrolídeos } \\
\text { (fortificação após a extração - método 5) }\end{array}$ & 1,20 \\
\hline $\begin{array}{c}\text { Mix de macrolídeos } \\
\text { (fortificação após a extração - método 7) }\end{array}$ & 3,00 \\
\hline
\end{tabular}

\section{Instrumentação LC-MS/MS}

A separação cromatográfica foi realizada utilizando um Cromatógrafo a Líquido de Alta Eficiência, modelo Prominence (Shimadzu, Kyoto, Japão) com módulo de aquecimento para coluna cromatográfica e dispositivo de resfriamento de amostra. A separação das substâncias foi realizada com coluna cromatográfica Pursuit ${ }^{\circledR}$ C18 (Agilent Technologies, Amsterdã, Holanda) (2,0 x 100 mm, 3 mm de tamanho de partícula) equipada com coluna de guarda Pursuit ${ }^{\circledR}$ C18 (Agilent Technologies, Amsterdã, Holanda) e temperatura do forno de $35{ }^{\circ} \mathrm{C}$. A fase móvel utilizada foi $0,1 \%(\mathrm{v} / \mathrm{v})$ de FOA em água (fase A), $0,1 \%(\mathrm{v} / \mathrm{v})$ de FOA em acetonitrila (fase B) e $0,1 \%(\mathrm{v} / \mathrm{v})$ de FOA em metanol (fase C). A eluição do gradiente foi: $41 \%$ de A e $59 \%$ de $\mathrm{C}$ até 4 min (fluxo de $0,25 \mathrm{~mL} \mathrm{~min}^{-1}$ ); $50 \%$ de $\mathrm{B}$ e $50 \%$ de $\mathrm{C}$

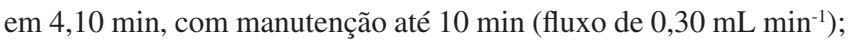
$41 \%$ de A de $59 \%$ de $\mathrm{C}$ em 10,10 min, com manutenção até 14 min (fluxo de $0,30 \mathrm{~mL} \mathrm{~min}^{-1}$ ) e restabelecimento do gradiente inicial com $41 \%$ de A de 59\% de C em 14,10 min, com manutenção até 16 min (fluxo de $0,25 \mathrm{~mL} \mathrm{~min}^{-1}$ ), resultando em uma corrida total de $16 \mathrm{~min}$. $\mathrm{O}$ volume de injeção foi de $25 \mu \mathrm{L}$ e as injeções foram efetuadas em duplicata. A detecção foi realizada em espectrômetro de massas sequencial triplo quadrupolo API 5000 (Applied Biosystems/MDS Sciex, CA, EUA), com interface eletrospray trabalhando no modo de ionização positivo. Os parâmetros espectrométricos foram: temperatura da fonte TurboIonSpray $=550{ }^{\circ} \mathrm{C}$; voltagem do potencial 
de entrada $=10 \mathrm{~V}$; voltagem do ionspray $=5000 \mathrm{~V}$; voltagem do detector $=2300 \mathrm{~V}$; voltagem do defletor $=-100 \mathrm{~V}$. Nitrogênio foi aplicado como gás de nebulização (50 psi), secagem (55 psi), cortina (10 psi) e colisão (4 psi). O dwell time foi definido como $30 \mathrm{~ms}$. A avaliação dos dados foi realizada com auxílio do software Analyst ${ }^{\circledR}$ V1.4.2. As condições de monitoramento de reações múltiplas (MRM) encontram-se descritas na Tabela 2.

\section{Métodos para extração das amostras}

Fortificação das amostras antes e após o processo de extração para avaliação da recuperação

A fortificação inicial da amostra seguiu o mesmo processo em todos os métodos. Pesou-se $2 \mathrm{~g}$ da amostra em 4 tubos de centrífuga de $50 \mathrm{~mL}$ distintos, os quais foram identificados como amostra controle conforme fortificada com padrão interno (ACCPI), amostra controle não conforme com fortificação no final (ACNCFF), amostra controle não conforme 1 ( $\mathrm{ACNC} 1)$ e amostra controle não conforme 2 (ACNC2). Um tubo de centrífuga de $50 \mathrm{~mL}$ para avaliação dos reagentes foi identificado como amostra controle branco de reagentes (ACBR). Aos tubos da ACBR e da ACNCFF foram adicionados $100 \mu \mathrm{L}$ de $\mathrm{MeOH}$. A ACCPI foi fortificada com $75 \mu \mathrm{L}$ de $\mathrm{MeOH}$ e $25 \mu \mathrm{L}$ da solução intermediária do padrão interno (ROX) a $2 \mu \mathrm{g} \mathrm{mL} \mathrm{m}^{-1}$. A ACNC1 e ACNC2 foram fortificadas com $50 \mu \mathrm{L}$ de $\mathrm{MeOH}, 25 \mu \mathrm{L}$ da solução intermediária do padrão interno e $25 \mu \mathrm{L}$ da solução intermediária do mix de macrolídeos para fortificação da amostra no início do processo de extração a $2 \mu \mathrm{g} \mathrm{mL} \mathrm{m}^{-1}$. Após agitação em vortex por $10 \mathrm{~s}$ os tubos foram mantidos em repouso por $10 \mathrm{~min}$. Métodos distintos de extração foram testados (métodos 1 a 8). Após os processos de extração, retirou-se $250 \mu \mathrm{L}$ de sobrenadante de cada um dos tubos, transferiu-se para tubos de centrífuga de $15 \mathrm{~mL}$ e evaporou-se até a secura sob fluxo de $\mathrm{N}_{2}$, à temperatura máxima de $47^{\circ} \mathrm{C}$. Ressuspendeu-se o extrato seco da ACBR, ACCPI, ACNC1 e ACNC2 com $1 \mathrm{~mL}$ de $\mathrm{MeOH}: \mathrm{H}_{2} \mathrm{O}(65: 35$, v/v), agitou-se em vortex por $15 \mathrm{~s}$ e filtrou-se a solução com filtro de PVDF de $0,22 \mu \mathrm{m}$ para um vial. Nos métodos identificados como 1, 2, 3, 4, 6 e 8, paralelamente, ressuspendeu-se o extrato seco da ACNCFF com $1 \mathrm{~mL}$ da solução padrão intermediária com mix de macrolídeos para fortificação da amostra após processo de extração, agitou-se em vortex por $15 \mathrm{~s} \mathrm{e}$ filtrou-se a solução com filtro de PVDF de $0,22 \mu \mathrm{m}$ para um vial. O mesmo procedimento foi adotado para os extratos secos da ACNCFF dos métodos 5 e 7, entretanto a fortificação da mesma foi realizada com $1 \mathrm{~mL}$ das soluções padrões intermediárias com mix de macrolídeos para fortificação da amostra após o processo de extração com as concentrações especificas para esses métodos.

\section{Procedimentos de extração}

Os métodos descritos como 1, 2, 3, 4 e 8 foram baseados em ensaios desenvolvidos previamente no laboratório, ${ }^{19-23}$ enquanto os

Tabela 2. Condições de fragmentação para identificação dos resíduos de macrolídeos por LC-MS/MS

\begin{tabular}{|c|c|c|c|c|c|c|c|}
\hline Substância & Íon precursor & $\begin{array}{c}\text { Massa } \\
\text { monoisotópica } \\
\text { (u) }\end{array}$ & $\begin{array}{l}\text { Íon precursor } \\
\qquad(\mathrm{m} / \mathrm{z})\end{array}$ & $\begin{array}{l}\text { Íon produto } \\
(\mathrm{m} / \mathrm{z})\end{array}$ & $\mathrm{DP}^{\mathrm{b}}$ & $\mathrm{CE}^{\mathrm{c}}$ & $\mathrm{CXP}^{\mathrm{d}}$ \\
\hline & & & & 158,2 & & 35 & 16 \\
\hline \multirow[t]{3}{*}{ CLA } & {$[\mathrm{CLA}+\mathrm{H}]^{+}$} & 748,500 & 748,5 & 590,2 & 146 & 25 & 20 \\
\hline & & & & 116,1 & & 49 & 16 \\
\hline & & & & 158,2 & & 41 & 14 \\
\hline \multirow[t]{3}{*}{ ERI } & {$[\mathrm{ERI}+\mathrm{H}]^{+}$} & 733,924 & 734,5 & 576,2 & 171 & 23 & 18 \\
\hline & & & & 116,0 & & 51 & 14 \\
\hline & & & & 174,0 & & 29 & 30 \\
\hline \multirow[t]{3}{*}{ ESPI } & {$[\mathrm{ESPI}+2 \mathrm{H}]^{2+}$} & 843,050 & 422,3 & 144,9 & 126 & 19 & 22 \\
\hline & & & & 540,2 & & 15 & 10 \\
\hline & & & & 158,2 & & 35 & 20 \\
\hline \multirow[t]{3}{*}{ OLE } & {$[\mathrm{OLE}+\mathrm{H}]^{+}$} & 687,856 & 688,3 & 544,4 & 136 & 21 & 18 \\
\hline & & & & 116,3 & & 55 & 14 \\
\hline & & & & 158,0 & & 21 & 22 \\
\hline \multirow[t]{3}{*}{ ROX } & {$[\mathrm{ROX}+2 \mathrm{H}]^{2+}$} & 837,043 & 419,4 & 116,0 & 86 & 33 & 20 \\
\hline & & & & 573,5 & & 13 & 18 \\
\hline & & & & 174,1 & & 49 & 18 \\
\hline \multirow[t]{3}{*}{ TIL } & {$[\mathrm{TIL}+\mathrm{H}]^{+}$} & 916,097 & 916,6 & 772,4 & 226 & 39 & 24 \\
\hline & & & & 156,1 & & 61 & 22 \\
\hline & & & & 174,0 & & 33 & 22 \\
\hline \multirow[t]{3}{*}{ TILM } & {$[\mathrm{TILM}+2 \mathrm{H}]^{2+}$} & 868,566 & 435,3 & 695,6 & 106 & 19 & 22 \\
\hline & & & & 126,1 & & 49 & 26 \\
\hline & & & & 158,1 & & 37 & 16 \\
\hline \multirow[t]{2}{*}{ TROL } & [TROL-ACETIL $]^{+}$ & 813,966 & 772,4 & 586,2 & 146 & 25 & 20 \\
\hline & & & & 116,0 & & 57 & 20 \\
\hline
\end{tabular}

a: Considerando as massas atômicas exatas dos isótopos mais abundantes; b: Declustering Potential (V); c: Collision Energy (eV); d: Collision Exit Potential (V). 
métodos 5, 6 e 7 basearam-se em métodos descritos por diversos autores, selecionados após levantamento bibliográfico. ${ }^{18,7,24}$

\section{Método 1: ACN}

Adicionou-se a todos os tubos duas porções de $4 \mathrm{~mL}$ de ACN, com agitação por 1 min no agitador múltiplo após a adição de cada porção. Agitou-se os tubos na mesa agitadora por $20 \mathrm{~min}$, em velocidade de $240 \mathrm{rpm}$, e centrifugou-se a $4{ }^{\circ} \mathrm{C}, 5000 \mathrm{rpm}$, por $5 \mathrm{~min}$.

Método 2: $\mathrm{ACN}+0,2 \mathrm{~g}$ de $\mathrm{NaOAc}+0,8 \mathrm{~g}$ de $\mathrm{MgSO}_{4}(\mathrm{QuEChERS})$

Adicionou-se a todos os tubos duas porções de $4 \mathrm{~mL}$ de $\mathrm{ACN}$, com agitação por 1 min no agitador múltiplo após a adição de cada porção. Adicionou-se a cada tubo $0,2 \mathrm{~g}$ de $\mathrm{NaOAc}$ e $0,8 \mathrm{~g}$ de $\mathrm{MgSO}_{4}$. Agitou-se por $1 \mathrm{~min}$ no agitador múltiplo e centrifugou-se a $4{ }^{\circ} \mathrm{C}$, $5000 \mathrm{rpm}$, por $5 \mathrm{~min}$.

Método 3: 0,1\% (v/v) de FOA em ACN + 0,2 g de NaOAC + 0,8 g de $\mathrm{MgSO}_{4}(\mathrm{QuEChERS})$

Adicionou-se a todos os tubos duas porções de $4 \mathrm{~mL}$ de $0,1 \%$ (v/v) de FOA em ACN, com agitação por 1 min no agitador múltiplo após a adição de cada porção. Adicionou-se a cada tubo $0,2 \mathrm{~g}$ de $\mathrm{NaOAc}$ e $0,8 \mathrm{~g}$ de $\mathrm{MgSO}_{4}$. Agitou-se por $1 \mathrm{~min}$ no agitador múltiplo e centrifugou-se a $4{ }^{\circ} \mathrm{C}, 5000 \mathrm{rpm}$, por $5 \mathrm{~min}$.

Método 4: $\mathrm{ACN}+0,2 \mathrm{~g}$ de $\mathrm{NaOAC}+0,8 \mathrm{~g}$ de $\mathrm{MgSO}_{4}+200 \mathrm{mg}$ de PSA (QuEChERS)

Adicionou-se a todos os tubos duas porções de $4 \mathrm{~mL}$ de ACN, com agitação por 1 min no agitador múltiplo após a adição de cada porção. Adicionou-se a cada tubo $0,2 \mathrm{~g}$ de $\mathrm{NaOAc}$ e $0,8 \mathrm{~g}$ de $\mathrm{MgSO}_{4}$. Agitou-se os tubos na mesa agitadora por $20 \mathrm{~min}$, em velocidade de $240 \mathrm{rpm}$, e centrifugou-se a $4{ }^{\circ} \mathrm{C}, 5000 \mathrm{rpm}$, por $5 \mathrm{~min}$. Para a limpeza dos extratos, de cada um dos tubos transferiu-se $2 \mathrm{~mL}$ do sobrenadante para tubos de centrífuga de $15 \mathrm{~mL}$ distintos contendo $200 \mathrm{mg}$ de PSA. Agitou-se por 1 min no agitador múltiplo e centrifugou-se a $4{ }^{\circ} \mathrm{C}, 5000 \mathrm{rpm}$ por $5 \mathrm{~min}$.

\section{Método 5: $1 \%(\mathrm{v} / \mathrm{v})$ de FOA em ACN}

Adicionou-se $2 \mathrm{~mL}$ de $\mathrm{H}_{2} \mathrm{O}$ aos tubos e agitou-se por $15 \mathrm{~s}$. Posteriormente adicionou-se a todos os tubos duas porções de $4 \mathrm{~mL}$ de $1 \%$ (v/v) de FOA em ACN, com agitação por 1 min no agitador múltiplo após a adição de cada porção. Agitou-se os tubos na mesa agitadora por $20 \mathrm{~min}$, em velocidade de $240 \mathrm{rpm}$, e centrifugou-se a $4{ }^{\circ} \mathrm{C}, 5000 \mathrm{rpm}$, por $5 \mathrm{~min}$

Método 6: $1 \%(v / v)$ de HOAc em ACN + 0,2 g de NaOAc + 0,8 g de $\mathrm{MgSO}_{4}(\mathrm{QuEChERS})$

Adicionou-se a todos os tubos duas porções de $4 \mathrm{~mL}$ de $1 \%(\mathrm{v} / \mathrm{v})$ de HOAc em ACN, com agitação por 1 min no agitador múltiplo após a adição de cada porção. Adicionou-se a cada tubo $0,2 \mathrm{~g}$ de $\mathrm{NaOAce} \mathrm{e} 0,8 \mathrm{~g}$ de $\mathrm{MgSO}_{4}$. Agitou-se por $1 \mathrm{~min}$ no agitador múltiplo e centrifugou-se a $4{ }^{\circ} \mathrm{C}, 5000 \mathrm{rpm}$, por $5 \mathrm{~min}$.

Método 7: 1\% (v/v) de HOAc em $\mathrm{H}_{2} \mathrm{O}: \mathrm{ACN}(16: 84, v / v)+2,4 \mathrm{~g}$ de $\mathrm{Na}_{2} \mathrm{SO}_{4}+0,58 \mathrm{~g}$ de $\mathrm{NaOAc}$ (QuEChERS)

Adicionou-se a todos os tubos duas porções de $2 \mathrm{~mL}$ de $1 \%(\mathrm{v} / \mathrm{v})$ de $\mathrm{HOAc}$ em $\mathrm{H}_{2} \mathrm{O}: \mathrm{ACN}$ (16:84, v/v), com agitação por 1 min no agitador múltiplo após a adição de cada porção. Adicionou-se a cada tubo 2,4 $\mathrm{g}$ de $\mathrm{Na}_{2} \mathrm{SO}_{4}$ e $0,58 \mathrm{~g}$ de NaOAc anidro. Agitou-se por $1 \mathrm{~min}$ no agitador múltiplo e centrifugou-se a $4{ }^{\circ} \mathrm{C}, 5000 \mathrm{rpm}$, por $5 \mathrm{~min}$.

Método 8: $\mathrm{ACN}+0,8 \mathrm{~g}$ de $\mathrm{Na}_{2} \mathrm{SO}_{4}+0,2 \mathrm{~g}$ de $\mathrm{NaCl}+0,4 \mathrm{~g}$ de $\mathrm{K}_{2} \mathrm{CO}_{3}(\mathrm{QuEChERS})$

Adicionou-se a todos os tubos duas porções de $4 \mathrm{~mL}$ de ACN, com agitação por 1 min no agitador múltiplo após a adição de cada porção. Adicionou-se a cada tubo $0,8 \mathrm{~g}$ de $\mathrm{Na}_{2} \mathrm{SO}_{4}, 0,2 \mathrm{~g}$ de $\mathrm{NaCl}$ e $0,4 \mathrm{~g}$ de $\mathrm{K}_{2} \mathrm{CO}_{3}$. Agitou-se por 1 min no agitador múltiplo e centrifugou-se a $4{ }^{\circ} \mathrm{C}, 5000 \mathrm{rpm}$, por $5 \mathrm{~min}$.

\section{Avaliação dos métodos}

Os métodos com melhores recuperações globais (Rglobal) dos analitos e menores desvios padrões relativos (RSD) foram selecionados. Para os cálculos da Rglobal e do RSD, primeiramente calculou-se os desvios padrões relativos entre as injeções (equação 1) e a recuperação (equação 2) de cada substância.

$$
\operatorname{RSDinj}(\%)=\frac{s\left(1^{\mathrm{a}} \text { e } 2^{\mathrm{a}} \text { injeção }\right)}{\bar{X}\left(1^{\mathrm{a}} \text { e } 2^{\mathrm{a}} \text { injeção }\right)} \times 100
$$

onde: s ( $1^{\mathrm{a}}$ e $2^{\mathrm{a}}$ injeção) é o desvio padrão entre a área obtida na integração do pico da substância na primeira injeção da ACNC (ou ACNCFF) e a área obtida na integração do pico da substância na segunda injeção da ACNC (ou ACNCFF); $\bar{X}$ ( $1^{\mathrm{a}}$ e $2^{\mathrm{a}}$ injeção) é a média entre a área obtida na integração do pico da substância na primeira injeção da ACNC (ou ACNCFF) e a área obtida na integração do pico da substância na segunda injeção da ACNC (ou ACNCFF).

$$
R(\%)=\frac{\bar{X} \operatorname{ACNC}\left(1^{\mathrm{a}} \text { e } 2^{\mathrm{a}} \text { injeção }\right)}{\bar{X} \operatorname{ACNCFF}\left(1^{\mathrm{a}} \text { e } 2^{\mathrm{a}} \text { injeção }\right)} \times 100
$$

onde: $\bar{X}$ ACNC ( $1^{\mathrm{a}}$ e $2^{\mathrm{a}}$ injeção) é a média entre a área obtida na integração do pico da substância na primeira injeção da ACNC1 (ou ACNC2) e a área obtida na integração do pico da substância na segunda injeção da $\mathrm{ACNC} 1$ (ou ACNC2); $\bar{X} \operatorname{ACNCFF}\left(1^{\mathrm{a}}\right.$ e $2^{\mathrm{a}}$ injeção) é a média entre a área obtida na integração do pico da substância na primeira injeção da $\mathrm{ACNCFF}$ e a área obtida na integração do pico da substância na segunda injeção da ACNCFF.

As Rglobal (equação 3) e os RSD (equação 4) foram calculados segundo as equações 3 e 4 :

$$
\begin{gathered}
\operatorname{Rglobal}(\%)=\bar{X} \mathrm{R} \% \text { ACNC1 e ACNC2 } \\
R S D(\%)=\frac{s(\mathrm{R} \% \text { ACNC1 e } \mathrm{R} \% \text { ACNC } 2)}{\text { Rglobal }} \times 100
\end{gathered}
$$

onde: $\bar{X} \mathrm{R} \%$ ACNC1 e ACNC2 é a média entre a recuperação da $\mathrm{ACNC} 1$ e da $\mathrm{ACNC} 2$; s ( $\mathrm{R} \% \mathrm{ACNC} 1$ e $\mathrm{R} \% \mathrm{ACNC} 2)$ é o desvio padrão entre a recuperação da $\mathrm{ACNC} 1$ e da ACNC2.

\section{RESULTADOS E DISCUSSÃO}

\section{Avaliação comparativa dos métodos de extração}

Os alimentos infantis industrializados (sopas) à base de carnes são matrizes complexas, com alto teor de proteínas, lipídeos e também de carboidratos quando legumes estão presentes na formulação. Portanto, são necessárias etapas de limpeza das amostras antes da identificação dos analitos por LC-MS/MS. Os métodos se diferenciaram basicamente pelos solventes e sais utilizados para a extração, os quais estão descritos na Tabela 3 .

A ACN foi empregada, pura ou em mistura, em todos os métodos por ser reconhecidamente um solvente utilizado para a precipitação de proteínas. ${ }^{19}$ As Rglobal e os RSD dos métodos de 1 a 8 encontram-se nas Figuras 2 e 3 respectivamente.

O conhecimento das propriedades físico-químicas das moléculas é fundamental para prever e explicar seus comportamentos frente às 
Tabela 3. Solventes e sais empregados em cada método de extração e limpeza do extrato

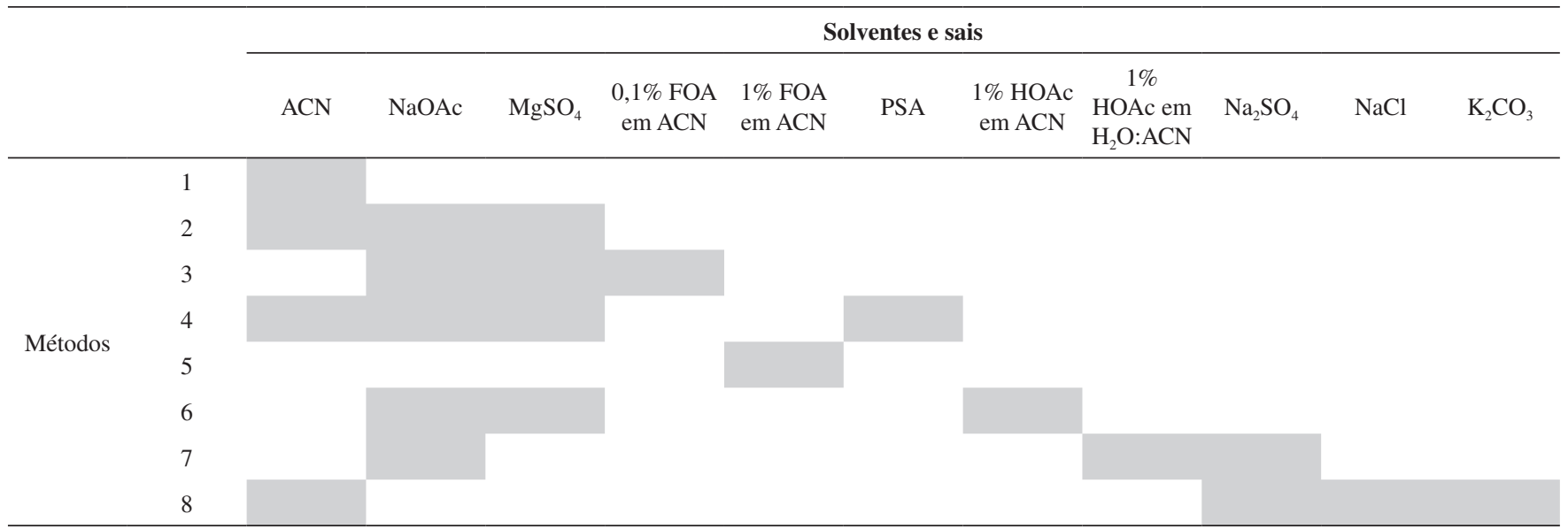

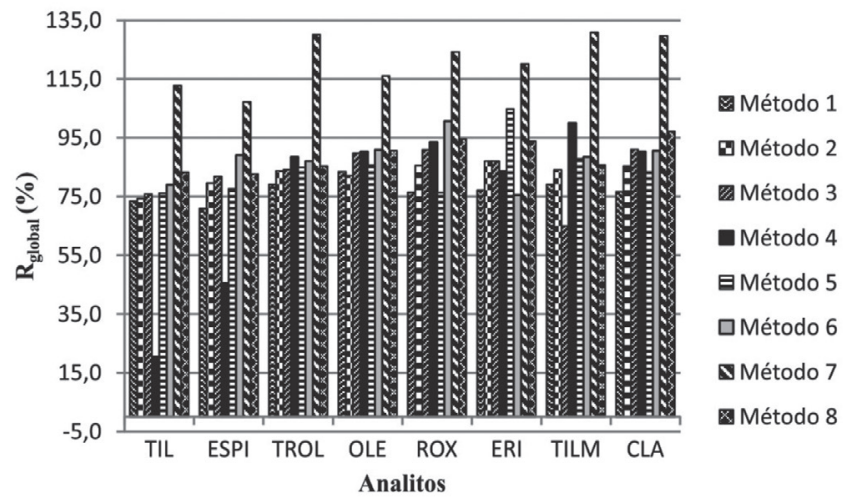

Figura 2. Rglobal(\%) dos analitos nos métodos 1, 2, 3, 4, 5, 6, 7 e 8

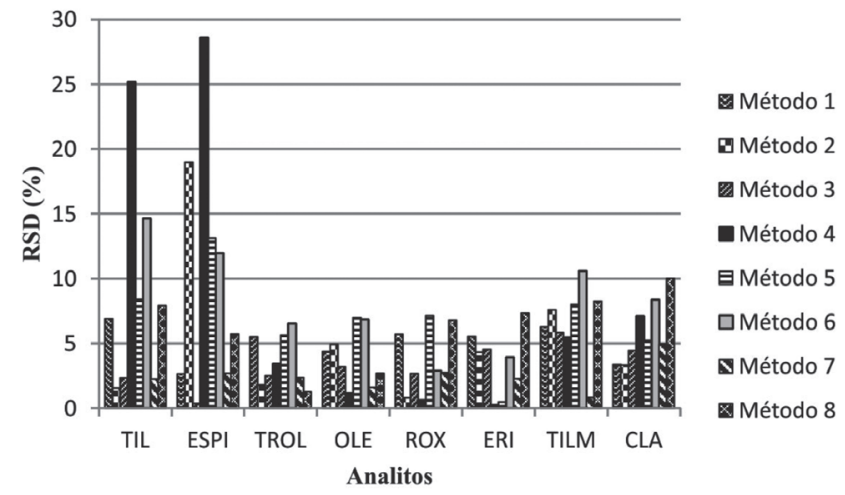

Figura 3. Desvio padrão relativo, $R S D(\%)$, entre ACNC1 e ACNC2 nos métodos 1, 2, 3, 4, 5, 6, 7 e 8

diferentes condições de extração. Quimicamente, os macrolídeos apresentam em geral $\mathrm{p} K$ a entre 7,1 e 9,9, tendo propriedades de bases fracas lipofílicas e alguns são sensíveis ao $\mathrm{pH}$ baixo sofrendo degradação em condições ácidas. ${ }^{10}$ Embora os valores de $\mathrm{p} K \mathrm{a}>7$ e do logaritmo do coeficiente de partição $\log P>2$ dos macrolídeos indiquem que a extração dessas substâncias em meio ácido não seja favorecida, por estarem na forma de cátions hidrofílicos, devido à protonação dos grupamentos amino (Figura 2), alguns autores empregaram com sucesso acetonitrila ou misturas de acetonitrila e água adicionadas de ácido fórmico ou ácido acético, com ou sem adição de sais, para extrair esses antimicrobianos de alimentos infantis. ${ }^{7,18,24}$ Os ácidos são geralmente utilizados para competir com o complexo analito-proteína, resultando em um aumento da recuperação. Considerando que alguns dos analitos pesquisados ligam-se fortemente a proteínas, tais como a eritromicina (95\%) e roxitromicina $(96 \%)$, a utilização de solventes acidificados pode favorecer a recuperação. Entretanto, a presença de co-extrativos na fase orgânica obtida no processo de extração em meio ácido pode ocasionar um aumento do efeito de matriz, identificado muitas vezes pela pouca repetibilidade dos resultados de alíquotas verdadeiras ou de replicatas de injeção.

O método 1, que baseia sua extração unicamente na ACN, apresentou valores intermediários de Rglobal (71-83\%) e valores de RSD de no máximo $7 \%$. No método 5 , a acidificação da ACN com $1 \%$ (v/v) de FOA proporcionou um aumento da Rglobal para todos os analitos, com exceção da ROX, cuja recuperação manteve-se inalterada. Entretanto, ocorreu aumento nos RSD para TIL, OLE, ROX, TILM, CLA e ESPI, substância que apresentou considerável elevação do RSD, de $3 \%$ no método 1 para $13 \%$ no método 5 .

A partição de um analito entre uma fase aquosa e outra orgânica, com a adição de sais para promoção do efeito de salting-out, fundamento básico do método de extração QuEChERS, depende da estrutura da molécula, da distribuição das microespécies em função do $\mathrm{pH}$, a partir dos valores de $\mathrm{p} K$ a dos grupamentos ionizáveis, e das mudanças na solubilidade de acordo com a força iônica. Os perfis dos logaritmos dos coeficientes de distribuição $(\log D)$ versus valores de $\mathrm{pH}$ dos analitos estudados indicam que valores de $\log D>0$, correspondentes à lipofilia, com favorecimento da partição na fase orgânica, são alcançados a partir de pH 4,9 (TIL) a 7,1 (ESPI), sendo que para TROL $\log D>0$ para todo o intervalo de $\mathrm{pH} .{ }^{11}$ Isso indica que, exceto para a TROL, que independe do $\mathrm{pH}$, em valores de $\mathrm{pH}$ mais elevados a recuperação seria favorecida. A utilização dos sais secantes sulfato de magnésio e sulfato de sódio diminuem a quantidade de água na fase orgânica e os sais acetato de sódio, cloreto de sódio e carbonato de potássio promovem o efeito salting-out em diferentes valores de $\mathrm{pH}$, reduzindo a solubilidade dos analitos na fase aquosa, com a obtenção de maiores percentuais de recuperação na fase orgânica.

Ao se avaliar o método 2, cuja diferença para o método 1 é o emprego dos sais $\mathrm{NaOAc}$ e $\mathrm{MgSO}_{4}$, pode-se observar um aumento considerável na Rglobal dos analitos, exceto para OLE, cuja recuperação se manteve semelhante, e uma diminuição dos valores dos RSD para praticamente todos os analitos, com exceção da ESPI, cujo RSD atingiu o valor de $19 \%$. Por ter apresentado excelentes resultados foi proposta uma etapa de limpeza ao método 2 com emprego de PSA, dando origem ao método 4. Tal método, apesar da etapa de purificação, se mostrou inadequado, com a redução acentuada da Rglobal para TIL e ESPI, com grande aumento dos RSD para essas substâncias. TIL e ESPI, por serem os macrolídeos menos lipofílicos e mais polares dos estudados $(\log P 2,32$ e 2,5, respectivamente), sofreram 
maior retenção pelo PSA, conhecido como quelante de açúcares, ácidos graxos e outras substâncias polares de amostras complexas.

Ainda em comparação com o método 2, desenvolveu-se o método 3, cuja diferença foi a acidificação da ACN com 0,1\% (v/v) de FOA. Excelentes resultados foram obtidos tanto para os RSD quanto para as Rglobal, sendo a TILM o único analito a apresentar diminuição da Rglobal (65\%). Com relação ao método 7, baseado na extração com $1 \%$ (v/v) de $\mathrm{HOAc}$ em $\mathrm{H}_{2} \mathrm{O}: \mathrm{ACN}, \mathrm{NaOAc}$ e $\mathrm{Na}_{2} \mathrm{SO}_{4}$, o mesmo não se apresentou adequado, visto que as Rglobal ficaram acima de $107 \%$ para todas as substâncias analisadas, embora os RSD tenham apresentado valores $<5 \%$. Considerando que $\log P>0$ para todos os analitos pesquisados, a adição de água à acetonitrila não se fez necessária para uma extração eficiente desses antimicrobianos, e ainda promoveu a extração de co-extrativos polares responsáveis pelo efeito matriz identificado pelas recuperações acima de $100 \%$. O método 6, que também empregou HOAc, apresentou RSD acima de $10 \%$ para três substâncias (TIL, ESPI e TILM), demonstrando uma falta de reprodutibilidade entre as injeções das ACNC1 e ACNC2, também sendo considerado inadequado. O método 8, diferentemente dos demais, utilizou $\mathrm{NaCl}$ e $\mathrm{K}_{2} \mathrm{CO}_{3}$, além de $\mathrm{Na}_{2} \mathrm{SO}_{4}$ em seu procedimento de extração, alcançando altos valores de Rglobal (83-97\%) e baixos valores de RSD $(\leq 10 \%)$, se mostrando adequado para a análise de alimentos infantis. A adição de $\mathrm{K}_{2} \mathrm{CO}_{3}$ elevou o $\mathrm{pH}$ a valores próximos ou superiores aos $\mathrm{p} K \mathrm{a}$ dos analitos, favorecendo a partição das substâncias para a fase de acetonitrila. Com isso, os métodos 2, 3 e 8 foram selecionados para a repetição dos testes de extração (Figuras 4 e 5).

Após a realização de novos ensaios, observou-se que os métodos 2 e 8 apresentaram grandes variações entre o primeiro e o segundo dia de ensaio, com valores de RSD de até $16 \%$ (ROX) e de até $20 \%$ (TROL), respectivamente para os métodos 2 e 8 no segundo dia de ensaio e Rglobal (> 100\%) para as substâncias TIL, ESPI e ROX no segundo dia de ensaio no método 8 . O método 3 apresentou os melhores valores de Rglobal e RSD, além de menor variação entre o primeiro e segundo dia de ensaios realizados, exceto para ESPI e TILM, demonstrando melhor precisão. A Figura 6 exibe os cromatogramas de íons extraídos referentes às transições de quantificação da ROX e TROL em solução a $1,5 \mathrm{ng} \mathrm{mL}^{-1}$ (a, c) e na matriz fortificada (b, d) em concentração equivalente $\left(25 \mu \mathrm{g} \mathrm{kg}^{-1}\right)$ no método de extração 3 .

\section{CONCLUSÃO}

Um método qualitativo rápido para identificação de resíduos de macrolídeos em alimentos infantis por LC-MS/MS foi obtido. O preparo das amostras foi baseado no método QuEChERS com acetonitrila. O método de extração 3, empregando $0,1 \%$ (v/v) de FOA em

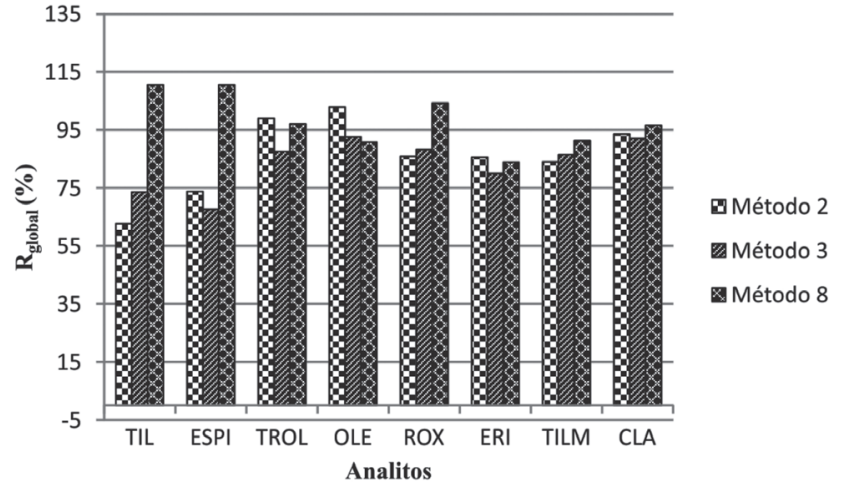

Figura 4. Rglobal(\%) dos analitos nos métodos 2, 3 e 8

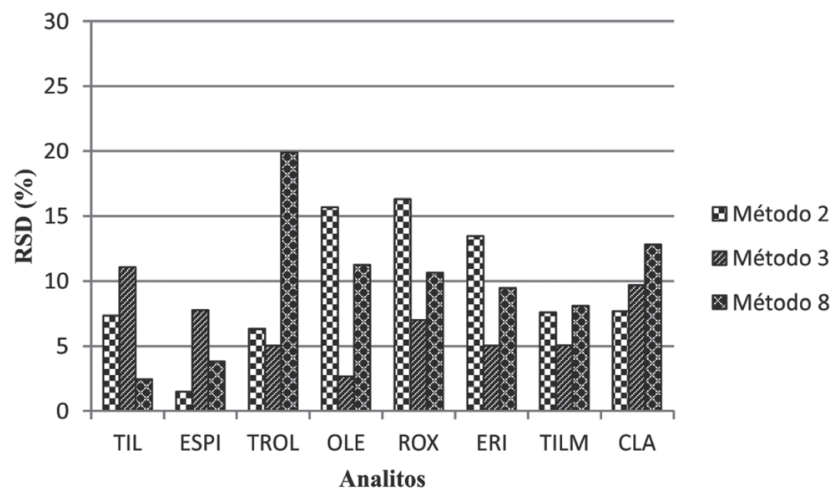

Figura 5. Desvio padrão relativo, $R S D(\%)$, entre ACNC1 e ACNC2 nos métodos 2,3 e 8

$\mathrm{ACN}, \mathrm{NaOAc}$ e $\mathrm{MgSO}_{4}$, se mostrou simples e efetivo, pois não foram necessárias etapas adicionais de limpeza. As recuperações e desvios padrões relativos apresentaram valores entre 65 e $93 \%$ e 0,4 e $11 \%$, respectivamente, para os analitos em questão em ensaios realizados em dois dias diferentes. Tais resultados sugerem que, após validado, o método selecionado, se aplicado à amostras de alimentos infantis, do tipo sopas, poderá subsidiar a avaliação da exposição de crianças e bebês aos resíduos dos medicamentos veterinários da classe dos macrolídeos em futuras ações de vigilância sanitária.

\section{AGRADECIMENTOS}

Ao Instituto Nacional de Controle de Qualidade em Saúde da Fundação Oswaldo Cruz (INCQS-FIOCRUZ) por todo o suporte.
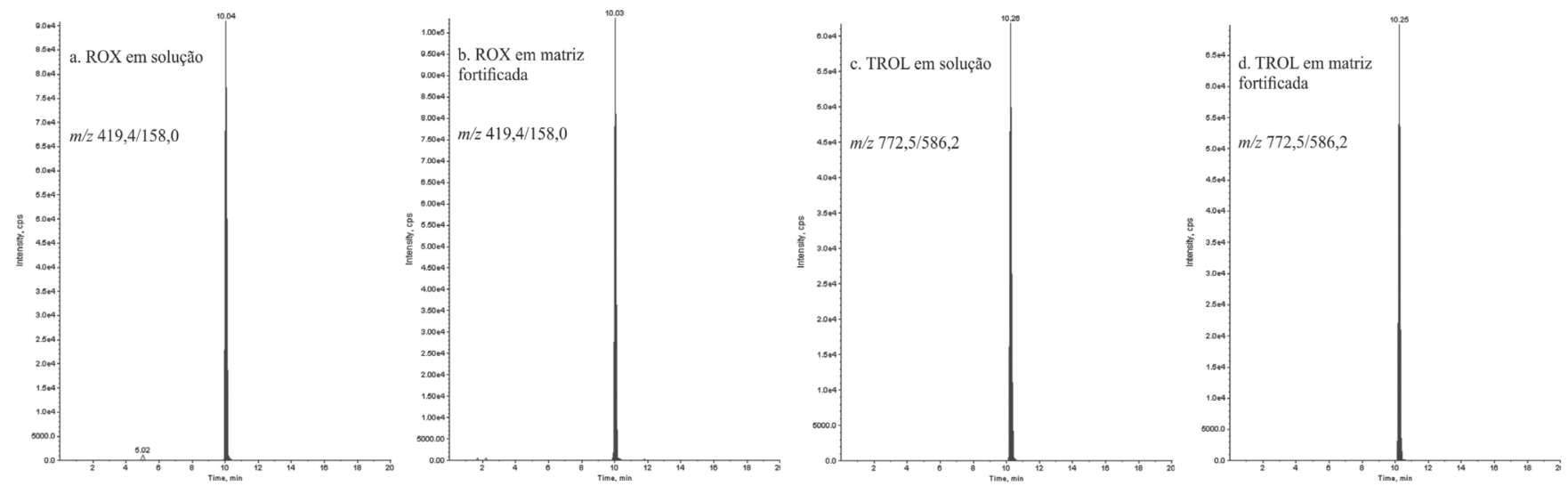

Figura 6. Cromatogramas de íons extraídos referentes às transições de quantificação da ROX e TROL em solução a 1,5 ng $\mathrm{mL}^{-1}$ (a, c) e na matriz fortificada (b, d) em concentração equivalente $\left(25 \mu \mathrm{gg}^{-1}\right)$ no método de extração 3 


\section{REFERÊNCIAS}

1. Spisso, B. F.; Nóbrega, A. W.; Marques, M. A. S.; Ciência \& Saúde Coletiva 2009, 14, 2091

2. Spinosa, H. S.; Palermo-Neto, J.; Górniak, S. L.; Medicamentos em Animais de Produção, $1^{\text {a }}$ ed., Guanabara Koogan: Rio de Janeiro, 2014.

3. Caballeto Torres, A. E. Em Temas de Higiene de los Alimentos; Sánchez, N. C., ed.; Ciencias Médicas: Ciudad de La Habana, 2008, cap. 22.

4. http://www.fao.org/fao-who-codexalimentarius/codex-texts/dbs/ vetdrugs/glossary/en/, acessada em outubro de 2018.

5. Baynes, R. E.; Dedonder, K.; Kissell, L.; Mzyk, D.; Marmulak, T.; Smith, G.; Tell, L.; Gehring, R.; Davis, J.; Riviere, J. E.; Food Chem. Toxicol. 2016, 88,112 .

6. Portaria n. 34, de 13 de janeiro de 1998. Aprova o Regulamento Técnico referente a Alimentos de Transição para Lactentes e Crianças de Primeira Infância, constante do anexo desta Portaria. Diário Oficial da União, Brasília, 16 de Janeiro de 1998.

7. Aguilera-Luiz, M. M.; Vidal, J. L. M.; Romero-González, R.; Frenich, A. G.; Food Chem. 2012, 132, 2171.

8. http://www.oie.int/fileadmin/Home/eng/Our_scientific_expertise/docs/ pdf/Eng_OIE_List_antimicrobials_May2015.pdf, acessada em outubro de 2018 .

9. http://apps.who.int/iris/bitstream/10665/255027/1/9789241512220-eng. pdf, acessada em acessada em outubro de 2018.

10. Sismotto, M.; Paschoal, J. A. R.; Reyes, F. G. R.; Quim. Nova 2013, 36, 449.

11. https://chemaxon.com/products/calculators-and-predictors\#logp_logd, acessada em outubro de 2018.
12. Rodriguez, E.; Moreno-Bondi, M. C.; Marazuela, M. D.; Food Chem. 2011, 127, 1354.

13. Blasco, C.; Torres, C. M.; Picó, Y.; TrAC, Trends Anal. Chem. 2007, 26, 895.

14. Rodriguez, E.; Moreno-Bondi, M. C.; Marazuela, M. D.; J. Chromatogr A 2008, 1209, 136.

15. Anastassiades, M.; Lehotay, S. J.; J. AOAC Int. 2003, 86, 412.

16. Prestes, O. D.; Friggi, C. A.; Adaime, M. B.; Zanella, R.; Quim. Nova 2009, 32, 1620.

17. Prestes, O. D; Adaime, M. B.; Zanella, R.; Scientia Chromatographica 2011, 3, 51 .

18. Gómez-Pérez, M. L.; Romero-González, R.; Vidal, J. L. M.; Frenich, A. G.; Talanta 2015, 131, 1 .

19. Spisso, B. F.; Ferreira, R. G.; Pereira, M. U.; Monteiro, M. A.; Cruz, T. A.; Costa, R. P.; Lima, A. M. B.; Nóbrega, A. W.; Anal. Chim. Acta 2010, 682, 82

20. Pereira, M. U.; Spisso, B. F.; Jacob, S. C.; Monteiro, M. A.; Ferreira, R. G.; Carlos, B. S.; Nóbrega, A. W.; Food Chem. 2016, 196, 130.

21. Santos, J. R. M. P.; Trabalho de conclusão de curso de especialização, Instituto Nacional de Controle de Qualidade em Saúde, Fundação Oswaldo Cruz, Brasil, 2015.

22. Melo, J. M. M. C.; Trabalho de conclusão de curso de especialização, Instituto Nacional de Controle de Qualidade em Saúde, Fundação Oswaldo Cruz, Brasil, 2015.

23. Costa, R. P.; Spisso, B. F.; Pereira, M. U.; Monteiro, M. A.; Ferreira, R. G.; Nóbrega, A. W.; J. Sep. Sci. 2015, 38, 3743.

24. Jia, W.; Chu, X.; Ling, Y; Huang, J.; Chang, J.; J. Chromatogr. A 2014, 1347,122 . 